\title{
Integration of Uncertain Ramp Area Aircraft Trajectories and Generation of Optimal Taxiway Schedules at Charlotte Douglas (CLT) Airport
}

\author{
William J. Coupe ${ }^{*}$ and Dejan Milutinović ${ }^{\dagger}$ \\ Jack Baskin School of Engineering, University of California, Santa Cruz, CA 95064, USA \\ Waqar Malik $\ddagger$ \\ University of California, Santa Cruz, NASA Ames Research Center, Moffett Field, CA 94035, USA \\ Yoon Jung $\S$ \\ NASA Ames Research Center, Moffett Field, CA 94035, USA
}

\begin{abstract}
The integration of aircraft maneuver characteristics into an optimal taxiway scheduling solution is challenging due to the uncertainties that are intrinsic to ramp area aircraft trajectories. To address the challenge, we build a stochastic model of ramp area aircraft trajectories that is used to generate a probabilistic measure of conflict within the Charlotte Douglas International Airport (CLT) ramp area. Parameters of the conflict distributions are estimated and passed to a Mixed Integer Linear Program that solves for an optimal taxiway schedule constrained to be conflict free in the presence of trajectory uncertainties. Here we extend our previous research by accounting for departing and arriving aircraft whereas our prior formulation only accounted for departing aircraft.
\end{abstract}

\section{Introduction}

Airport runways and taxiways have been identified as a bottleneck of the National Airspace System, and the major inhibiting factor for serving an increasing air traffic demand. In order to keep up with the increase of traffic density, new techniques are required to increase airport throughput while maintaining safe separation constraints. Since many airports operate at or close to their maximum capacity, an optimization of runway and taxiway operations is necessary. ${ }^{1}$ However, once their operations are improved by an optimal taxiway schedule, its execution ultimately depends on the ramp controllers who control gate push backs and ramp area aircraft maneuvers within the ramp area. ${ }^{2}$

Most of the previous taxiway scheduling research has focused on modeling an airport as a graph, i.e., a connected network, with aircraft travelling along the graph edges. In order to solve optimization problems on the graph authors have used genetic algorithms, ${ }^{3,4}$ Mixed Integer Linear Programs (MILPs), ${ }^{5}$ or hybrids of these. ${ }^{6,7}$ The MILP approach has been used in $8,9,10,11,12,13,14,15$ to optimize the routing and scheduling of airport surface traffic. The approach has been applied in ${ }^{16,17}$ where an optimization model is formulated for taxi scheduling at Dallas-Fort Worth International Airport (DFW). Similar work ${ }^{18}$ has formulated the problem to include uncertainties related to constraint satisfaction while uncertainties in aircraft taxiing has been considered in. ${ }^{19,20,21}$ Previous works, however, have only addressed maneuvers in the active movement area (i.e., taxiways and runways controlled by FAA air traffic control tower) and do not consider ramp areas. This research attempts to address the integration of a state-of-the-art optimal taxiway scheduler and ramp area aircraft maneuvers. ${ }^{22}$ To the best of our knowledge this research is the first attempt to address the taxi scheduler problem in the ramp area.

*AIAA Graduate Student Member, Graduate Student, Computer Engineering Department, UC Santa Cruz.

$\dagger$ Associate Professor, Computer Engineering Department, UC Santa Cruz

${ }^{\ddagger}$ Research Scientist, University Affiliated Research Center, MS 210-8, Moffett Field, CA 94035.

$\S$ AIAA senior member and Aerospace Engineer, NASA Ames Research Center, MS 210-6, Moffett Field, CA 94035. 
In this paper we develop a general methodology to integrate uncertain ramp area aircraft trajectories within the framework of an optimal taxiway scheduling problem. In our previous work $^{22}$ we designed a scaled down robot experiment to generate data that was used as input to a stochastic model of aircraft trajectories. Using the stochastic aircraft trajectories we computed a probabilistic measure of conflicts among departing aircraft within the DFW airport ramp area. While in this paper we apply a similar approach to the Charlotte Douglas International Airport (CLT) ramp area, here we extend our previous work by a simultaneous consideration of departing and arriving aircraft. Moreover, the layout of the CLT ramp area presents new challenges due to the unique geometric constraints and high density of aircraft.

The main difficulty in the integration of ramp area aircraft maneuvers into an optimal taxiway scheduling solution is in uncertainties of ramp area trajectories. Unlike aircraft maneuvers on taxiways, ramp area aircraft maneuvers are frequently not confined to well-defined trajectories. The shape and timing of the trajectories are subject to uncertainties resulting from pilots decisions as well as other factors involved in ramp area operations, which can impede an optimal taxiway schedule plan. To account for these uncertainties, we model the trajectories as stochastic processes. However, ramp area trajectory data that could be used to build maneuver models are not readily available mainly due to the lack of surveillance data in the ramp area.

To address the lack of data, we collect the critical data on how a human operator navigates an aircraft within the ramp area using an inexpensive scaled down wheeled robot experiment. We use e-puck robots ${ }^{23}$ controlled by a human operator to simulate the movement of aircraft from the gate to the taxiway spot and collect the trajectory data with a standard webcam. The data is then processed in MATLAB to provide a time series of position and heading angle measurements of the simulated aircraft. By using the robot experiments, we are in position to go beyond the limits imposed by the lack of surveillance data and are able to collect realistic data that is physical in nature and includes variabilities due to human pilots.

The collected data is critical because it is used to estimate parameters of our stochastic model of aircraft trajectories. The stochastic trajectories replicate the statistical properties of the simulated aircraft data and allow us to find optimal taxiway schedules that account for uncertainties generated from the presence of a human pilot. Finally, our work demonstrates the utility of spatiotemporal data that could be captured from ramp area surveillance equipment if it existed. It is unlikely that airports will invest in the surveillance system unless its usefulness to the efficiency of operations can be shown.

This paper is organized as follows. In section II we formulate the problem under consideration. In section III we provide information regarding specific operational procedures at the CLT airport. Then, in section IV we describe the methodology for our stochastic model of aircraft trajectories. We present data associated with the sampled trajectories and conflict distributions in section V. Next, in section VI we provide the mathematical formulation of the Mixed Integer Linear Program and in section VII we provide illustrative examples. In the last section VIII, we conclude with a discussion of our findings and provide directions for future work.

\section{Problem Formulation}

Departing aircraft $i$ is parked at the gate. Upon receiving the push back clearance, a tug (operated by ground crew) pushes back the aircraft from the gate. At the end of the push back procedure, the aircraft stops and the tug disengages. This stop period lasts for some time during which the pilot goes through a checklist and then starts the aircraft engine(s). When ready the pilot requests taxi approval, and after the approval, taxies the aircraft until arriving at time $t_{i}$ at the terminal node (P1), see Fig 1. During the departing maneuvers the duration of the trajectory, the transitions over the motion phases, and the trajectory path are determined by human operators and are stochastic in nature.

Arriving aircraft $i$ begins its trajectory at the initial node (P2), see Fig 1 . The location of the initial node and the terminal node define a boundary between the graph (blue and yellow structured roadways) and the ramp area. After being released into the ramp area at time $t_{i}$, the aircraft taxis to the assigned gate. During the arriving maneuvers the duration of the trajectory and the trajectory path are considered to be stochastic.

The uncertain nature of ramp area trajectories can impede upon an optimal schedule that is defined on the graph. It is possible to compute a feasible schedule on the graph where ramp area aircraft will have to slow down or stop along their route to avoid a loss of separation. In contrast, we consider computing an optimal schedule on the graph that anticipates the uncertainty in such a way that every aircraft can proceed 


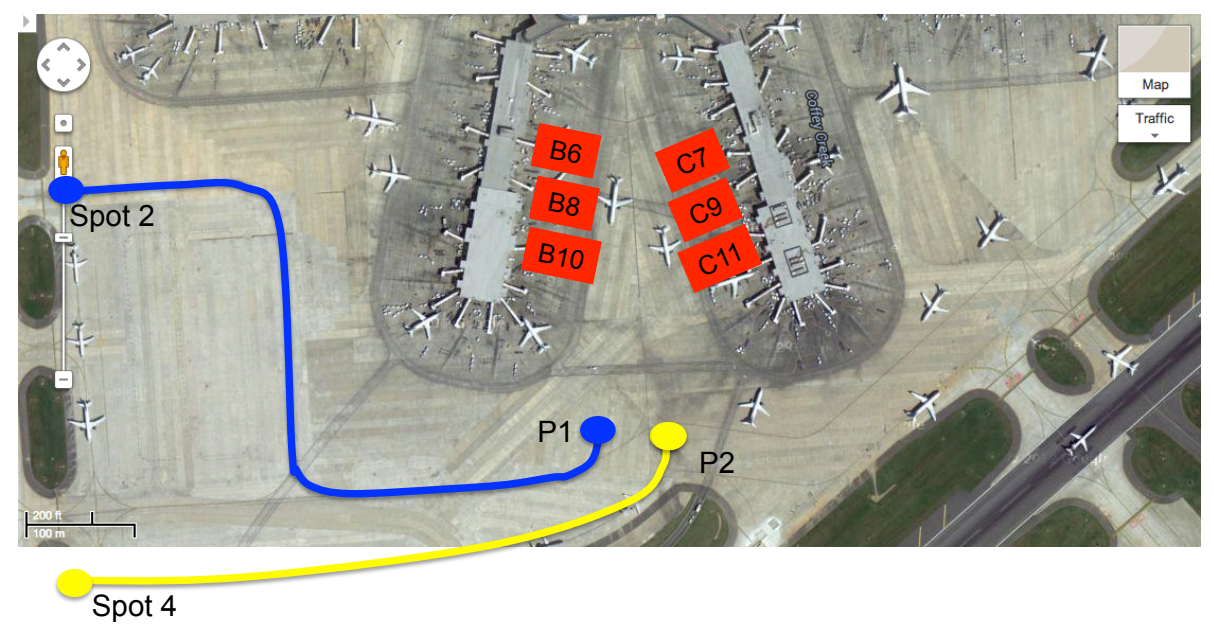

Figure 1. Center alley of the CLT airport. The gates under consideration are highlighted in red and include gates B6, B8, B10, C7, C9 and C11. Departing aircraft push back from their gate, enter into an uncertain stopped period, and then taxi to merge node P1. Arriving aircraft are released from merge node P2 and taxi to their assigned gate.

along their route without having to slow down or stop for other traffic.

Modeling trajectories as stochastic processes we compute a probabilistic measure of conflict among aircraft $i$ and $j$ defined by their relative schedule $t_{j}-t_{i}$. A conflict distribution is defined by a ratio of conflicts at a resolution of $1[s]$, see Fig 5 . We use the conflict distributions to calculate conservative conflict separation constraints that provide for safe separation in the presence of trajectory uncertainties.

We pass the conservative conflict separation constraints to an optimization problem on the graph that outputs a scheduled time $t_{i}$ for every aircraft. The optimal schedule is constrained to optimize the flow of traffic such that every aircraft $i$ can proceed along the route without stopping for other traffic.

For departure aircraft $i$ to proceed along the route without stopping, it is critical that the aircraft arrive at the terminal node P1 (boundary between the ramp area and the graph) at the scheduled time $t_{i}$. In order to aid ramp area controllers in meeting the scheduled times, we consider computing the feasible push back window for each departing aircraft. The push back window is defined by the earliest feasible push back time $t_{S}$ and latest feasible push back time $t_{F}$. Initiating the push back within the bounds $\left[t_{S}, t_{F}\right]$ ensures there exists a feasible trajectory that arrives at the terminal node $\mathrm{P} 1$ at the scheduled time $t_{i}$, as required by the optimal schedule.

\section{CLT Airport Surface Operations}

In this paper we consider the center alley of CLT airport with the B terminal on the left and C terminal on the right, see Fig. 1. The gates under analysis are highlighted in red and include B6, B8, B10, C7, C9 and $\mathrm{C} 11$. Each gate that we consider can either contain an aircraft ready for departure or receive an arrival aircraft if not currently occupied.

Aircraft departure trajectories begin by pushing back from their gate, entering an uncertain waiting period, followed by taxiing to spot 2 . The taxiway spot is used as a hold short node and aircraft are required to receive approval from controllers before transitioning between the ramp area and FAA taxiway. Along the taxi route to spot 2, departing aircraft travel over the ramp area merge node P1, see Fig. 1. The ramp area merge node $\mathrm{P} 1$ is introduced under an assumption that between merge node P1 and spot 2 there exists a well defined roadway for the aircraft to follow. Therefore, we assume that providing separation for departing aircraft along the route from their gate to merge node P1 ensures separation along the entire route from their gate to spot 2.

Aircraft arrival trajectories enter the ramp area at spot 4 and taxi to their assigned gates through the center alley. Arrival trajectories follow the well defined yellow structure from spot 4 to merge node P2. After arriving at P2, arrival aircraft are released into the center alley and taxi to their gate. We assume that 
providing the necessary separation for arriving aircraft along the route from merge node P2 to their gate ensures separation along the entire route from spot 4 to their gate.

\section{Methodology}

Data related to aircraft ramp area trajectories are not available or the existing data contains only the average value of trajectory duration. The available information is not sufficient to capture the evolution of individual aircraft trajectories. To account for this we use an inexpensive robot experiment setup where the movement of a Boeing 747-400 (Boeing 747) ${ }^{24}$ is simulated within the ramp area. ${ }^{22}$ Data from experiments are captured on video and processed in MATLAB to provide positions and orientations of simulated aircraft in time.

Collecting this data for multiple trajectories provides a distribution over the continuous interval of time that a trajectory can spend in the discrete states push back, stop, and taxi. ${ }^{22}$ We assume that the time spent in each discrete state is defined by a gamma distribution of the form

$$
X \sim \Gamma\left(k_{q}, \omega_{q}\right)
$$

with shape parameter $k_{q}$ and scale parameter $\omega_{q}$. Using the MATLAB function gamfit, we estimate parameters that fit the data from our robot experiments. The estimated gamma distributions for aircraft departure trajectories are depicted in Fig. 2.

Our data captures the influence of the human pilot and we use the collected data to fit parameters of our stochastic model of aircraft trajectories. Once the model is defined we use it to sample ${ }^{25,26}$ a large number of realistic trajectories. The sampled trajectories are used to build a probabilistic measure of conflict within the ramp area. After generating the measure of conflict, we calculate the necessary separation constraints in time among aircraft that ensure conflict free trajectories within the ramp area.

A single ramp area departure trajectory for aircraft $i$ is described by five discrete states $q, q=0 \ldots 4$. Each discrete state is defined by the continuous time evolution of the aircraft $i$ position and heading angle described by $x^{i}, y^{i}$ coordinates and $\theta^{i}$, respectively:

For $q=0$ (gate), $q=2$ (stop), $q=4$ (merge node $\mathrm{P} 1$ ):

$$
d x^{i}=0, d y^{i}=0, d \theta^{i}=0
$$

For $q=1$ (push back):

$$
d x^{i}=-v_{P}^{i} \cos \left(\theta^{i}\right) d t, d y^{i}=-v_{P}^{i} \sin \left(\theta^{i}\right) d t, d \theta^{i}=-\frac{v_{P}^{i}}{R^{i}} d t
$$

where $R^{i}$ is the radius of the circle of curvature that aircraft $i$ is pushing back along and $v_{P}^{i}$ is the push back velocity.

For $q=3$ (taxi):

$$
d x^{i}=v_{T}^{i} \cos \left(\theta^{i}\right) d t, d y^{i}=v_{T}^{i} \sin \left(\theta^{i}\right) d t, d \theta^{i}=\sigma^{i} d W^{i}
$$

where $d W^{i}$ is an increment of a unit intensity Wiener process, $\sigma^{i}$ is a scaling factor for the intensity of the variations in the heading angle $\theta^{i}$, and $v_{T}^{i}$ is the forward taxi velocity for aircraft $i$. In a similar fashion we can define the three discrete states $q$ for an arriving aircraft $i$ defined by the continuous time evolution:

For $q=0$ (merge node $\mathrm{P} 2), q=2$ (gate)

$$
d x^{i}=0, d y^{i}=0, d \theta^{i}=0
$$

For $q=1(\operatorname{taxi})$ :

$$
d x^{i}=v \cos \left(\theta^{i}\right) d t, d y^{i}=v \sin \left(\theta^{i}\right) d t, d \theta^{i}=\sigma^{i} d W^{i}
$$

Transitions between discrete states are considered to be stochastic. In order to simulate a single departure trajectory, we sample the times for states $q=1,2,3$ from the gamma distribution that was fitted to the robot 

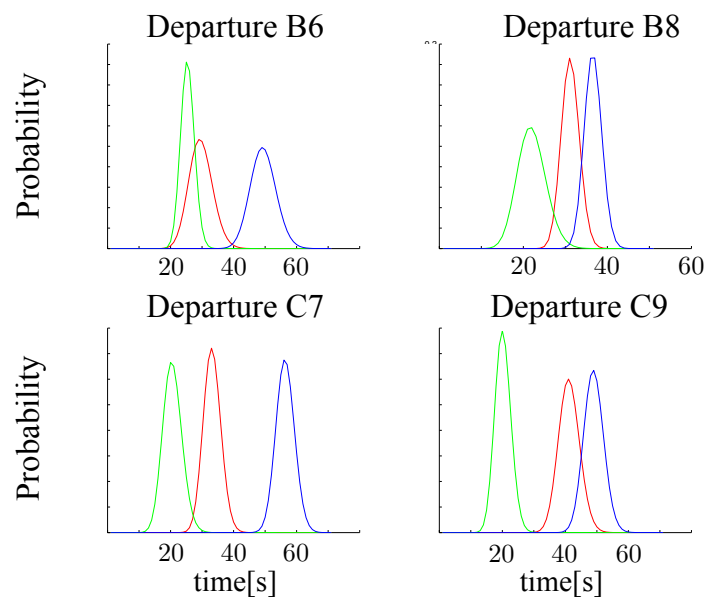

Departure C9
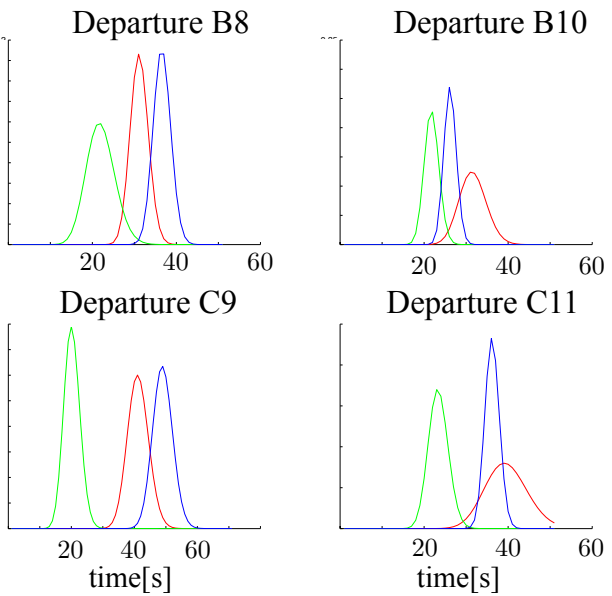

Figure 2. Distribution of time spent in discrete states for departing trajectories. The time spent in push back is shown in red, wait is shown in green, and taxi is shown in blue.

experiment data. Transitions between the states are defined by the values of the sampled times. In general, the times that we sample for the discrete states will never match exactly between two unique trajectories. For the set of successful samples, this temporal uncertainty will produce a distribution over the trajectory duration. This distribution in trajectory duration is directly influenced by the human operator.

In addition to the initial conditions and parameters we also define a terminal condition the trajectory sample must satisfy. Given that our trajectories are described by an uncontrolled stochastic processes, in general we do not expect the departure (arrival) samples to terminate at the merge node P1 (gate). However, if we sample enough departure (arrival) trajectories, we do expect for some samples to arrive at the merge node P1 (gate) as desired. Conditioning the trajectories to terminate within the goal region provides a set of feasible ramp area departure (arrival) trajectories that terminate (initiate) their trajectory at time $t_{i}$ at merge node P1 (P2).

After sampling trajectories we estimate the probability density function for trajectory duration of aircraft $i$ in the absence of any other aircraft in the ramp area. We refer to this type of distribution as natural since the aircraft is unimpeded. Since we are interested in the trajectory duration of departing aircraft $i$, we can enforce the terminal condition $t_{i}=0$, and this terminal condition provides a distribution for the push back time of feasible trajectories. In addition, enforcing this terminal condition in time provides us with a set of departing trajectories that all enter the FAA controlled taxiway via spot 2, see Fig. 1 , at the same time.

Using the family of trajectories defined by the natural distributions of aircraft $i$ and $j$, we generate a probabilistic measure of conflict. We compute the measure of conflict by fixing the terminal time of aircraft $i$ in time such that $t_{i}=0$. Next we fix the terminal time of aircraft $j$ in time, e.g. $t_{j}=-200$. Given the relative schedule defined by $t_{j}-t_{i}$, there exists a family of trajectories for both aircraft $i$ and $j$ that push back from their respective gate and taxi to merge node P1 as required.

For the relative schedule $t_{j}-t_{i}$, we sample a single trajectory from the family of trajectories for aircraft $i$ and $j$, measure their spatial proximity along the route, and provide a conflict flag if the aircraft lose spatial separation. If we continue this process of randomly sampling from the family of trajectories with fixed terminal times we compute a conflict ratio for the relative separation in time at the taxiway spot, see Algorithm 1. The fixed terminal times are considered for every whole second and the estimated conflict distributions provide a measure of conflict at a resolution of 1 second, see Fig. 5.

\section{Sampled Trajectories and Conflict Distributions}

Aircraft trajectories sampled from the stochastic model are shown in Fig. 3 and Fig. 4. These sampled trajectories are used to compute conflict distributions using Algorithm 1. The conflict distributions provide a conflict ratio among aircrafts $i$ and $j$ as a function of the difference between their merge node times, see Fig. 5. In this figure we assume departing aircraft $i$ always arrives at the merge node P1 at time $t_{i}=0$ and 


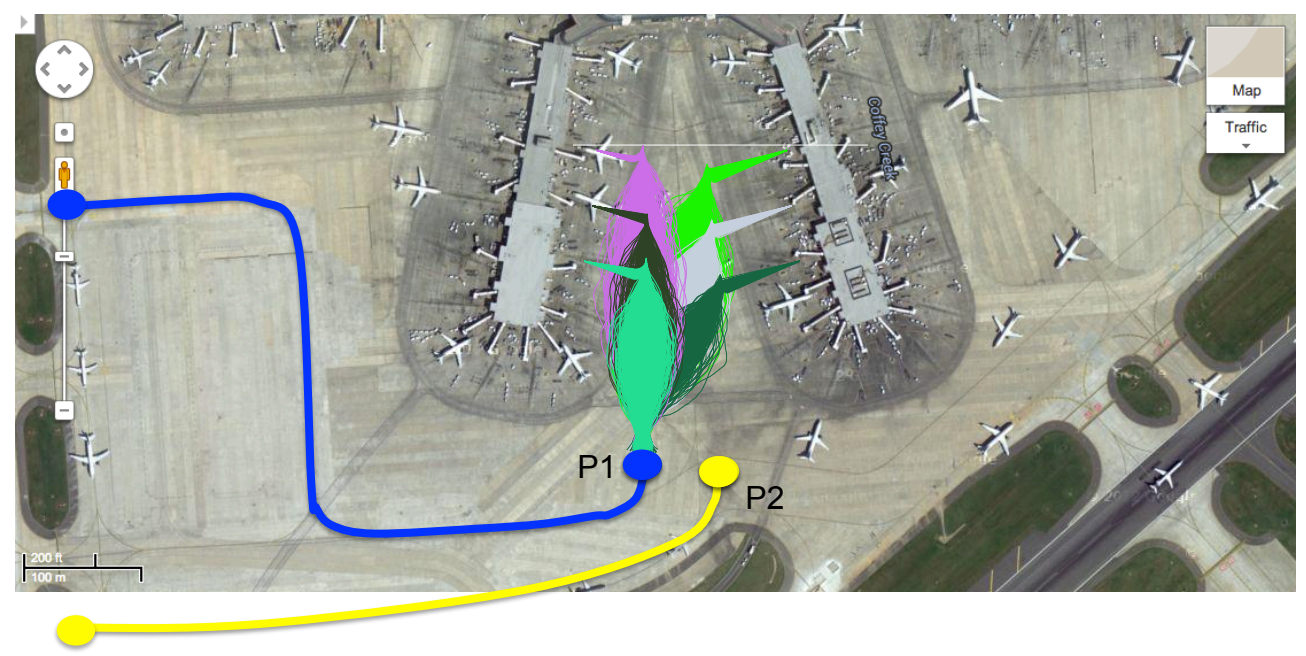

Figure 3. Sampled departure trajectories. For each gate, we generate a family of feasible departure trajectories. For each gate, the family of trajectories contains uncertainty within both the spatial path taken and trajectory duration.

departing aircraft $j$ arrives at the merge node defined by the value on the horizontal axis.

For conflicts that arise between two aircraft that travel through the same merge node(departure vs departure or arrival vs arrival conflicts) there exists a well defined sequence such that either aircraft $i$ comes prior to aircraft $j$ or vice versa. In these situations we simply separate the two aircraft at the merge node by the value $\delta_{i j}$ if aircraft $j$ follows aircraft $i$, else we separate the aircraft by the value $\delta_{j i}$, see left image of Fig. 5. By ensuring these minimum separation constraints we constrain the solution such that the conflict ratio among the two aircraft is zero.

For conflicts that arise between two aircraft that do not travel through the same merge node(departure vs arrival conflicts) there does not exist a well defined sequence, see right image of Fig. 5. In this figure we assume departing aircraft $i$ always arrives at the merge node P1 at time $t_{i}=0$ and arriving aircraft $j$ is released from the merge node P2 at the time defined by the value on the horizontal axis. Given that the values of $\Delta_{i j}^{L B}$ and $\Delta_{i j}^{U B}$ are both negative we can not simply select which separation constraint to use defined by the sequencing of aircraft at the merge node as we did before. To separate aircraft we should release the arriving aircraft to the left of the value $\Delta_{i j}^{L B}$ or to the right of the value $\Delta_{i j}^{U B}$.

\section{Mixed Integer Linear Program (MILP)}

Given a set of departing aircraft $i \in D$ available to push back from their gate at time $\alpha_{i}$ and a set of arriving aircraft $i \in A$ that are available to be released from node P2 into the ramp area at time $\beta_{i}$, we consider finding a sequence of merge node times $t_{i}$ that ensure conflict free trajectories. The optimal sequence of merge node times is defined as the schedule that minimizes the sum of aircraft hold time for both departing and arriving aircraft. The objective function is given by

$$
\min \left[\sum_{i \in D}\left(t_{i}-\left(\alpha_{i}+\left|t_{i}^{S 0}\right|\right)\right)+\sum_{i \in A}\left(t_{i}-\beta_{i}\right)\right]
$$

where $t_{i}^{S 0}$ is the earliest feasible push back time for departing aircraft $i$ such that the terminal time $t_{i}^{T}=0$ is enforced. For departing aircraft $i$, the difference between the scheduled terminal time $t_{i}$ and the earliest available push back time plus duration of the longest feasible trajectory, $\left(\alpha_{i}+\left|t_{S 0}^{i}\right|\right)$, describes the hold time for the individual aircraft. For arriving aircraft $i$, the difference between the scheduled time $t_{i}$ and the earliest available release time $\beta_{i}$ describes the hold time for the individual aircraft. Thus, within the objective function the total aircraft hold time for departing and arriving aircraft are given by the summations $\sum_{i \in D} t_{i}-\left(\alpha_{i}+\left|t_{S 0}^{i}\right|\right)$ and $\sum_{i \in A} t_{i}-\beta_{i}$ respectively. 


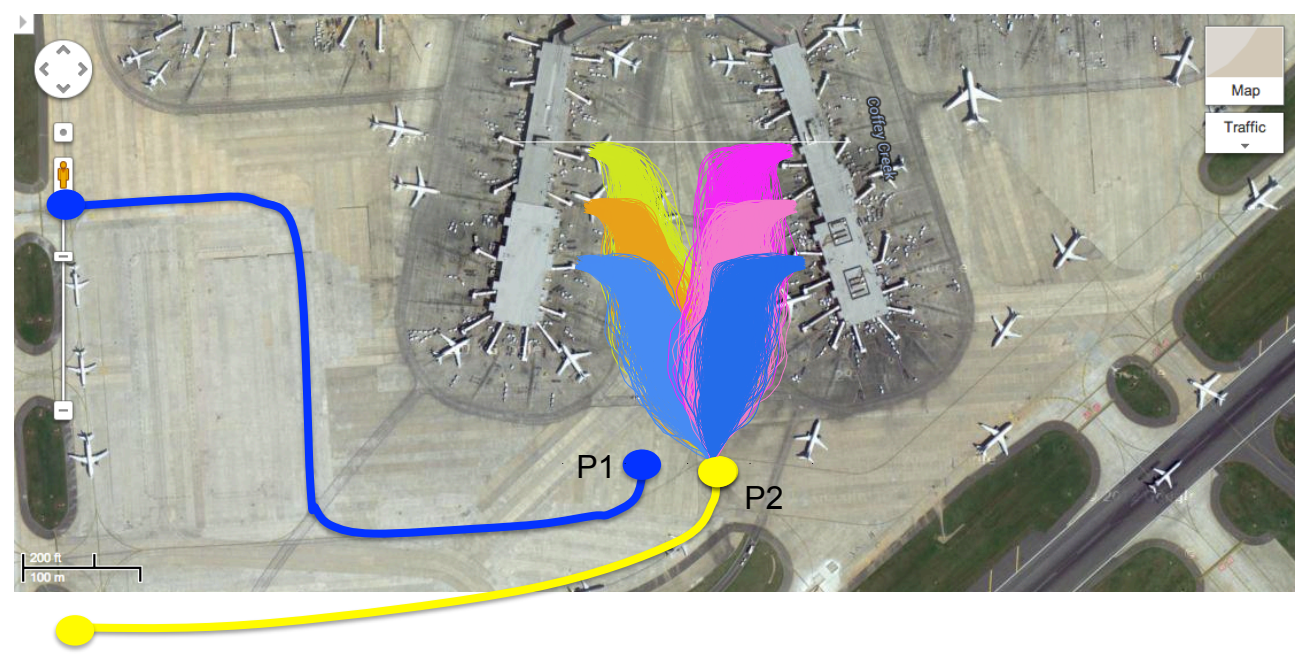

Figure 4. Sampled arrival trajectories. For each gate, we generate a family of feasible arrival trajectories. For each gate, the family of trajectories contains uncertainty within both the spatial path taken and trajectory duration.

For all departing aircraft $i \in D$ we introduce the constraint

$$
t_{i}-\left(\alpha_{i}+\left|t_{S 0}^{i}\right|\right) \geq 0 \quad \forall i \in D
$$

where this constraint ensures that for departing aircraft $i$ the scheduled terminal time $t_{i}$ at merge node P1 is greater than the earliest available push back time $\alpha_{i}$ plus the duration of the longest feasible trajectory $\left|t_{S 0}^{i}\right|$.

Similarly for all arriving aircraft $i \in A$ we have the constraint

$$
t_{i}-\beta_{i} \geq 0 \quad \forall i \in A
$$

which ensures that for arriving aircraft $i$ the scheduled time $t_{i}$ that we release the aircraft from merge node P2 into the ramp area is greater than the earliest time $\beta_{i}$ that the aircraft is available to be released. Constraints (8) and (9) in conjunction ensure that the hold time of any individual departing or arriving aircraft within the objective function is strictly positive, i.e., the minimum hold time for any aircraft $i$ is equal to zero.

For all departing aircraft $i, j \in D$ we introduce a sequencing constraint defined at merge node $\mathrm{P} 1$ given by

$$
z_{i j}+z_{j i}=1 \quad \forall i, j \in D
$$

where $z_{i j}$ is a binary variable that is 1 if departing aircraft $j$ follows departing aircraft $i$ at merge node $\mathrm{P} 1$, else $z_{i j}=0$. For all departing aircrafts $i, j \in D$ we have the separation constraint

$$
z_{i j}\left(t_{j}-t_{i}-\delta_{i j}\right) \geq 0 \quad \forall i, j \in D
$$

which ensures that if departing aircraft $j$ follows departing aircraft $i$ at merge node $\mathrm{P} 1$ they should be separated by a minimum of $\delta_{i j}$, else the constraint is automatically satisfied.

Similarly for all arriving aircraft $i, j \in A$ we introduce the sequencing constraint

$$
Z_{i j}^{*}+Z_{j i}^{*}=1 \quad \forall i, j \in A
$$

where $Z_{i j}^{*}$ is a binary variable that is 1 if arriving aircraft $j$ follows arriving aircraft $i$ at merge node P2, else $Z_{i j}^{*}=0$. For all arriving aircrafts $i, j \in A$ we have the separation constraint

$$
Z_{i j}^{*}\left(t_{j}-t_{i}-\delta_{i j}^{*}\right) \geq 0 \quad \forall i, j \in A
$$




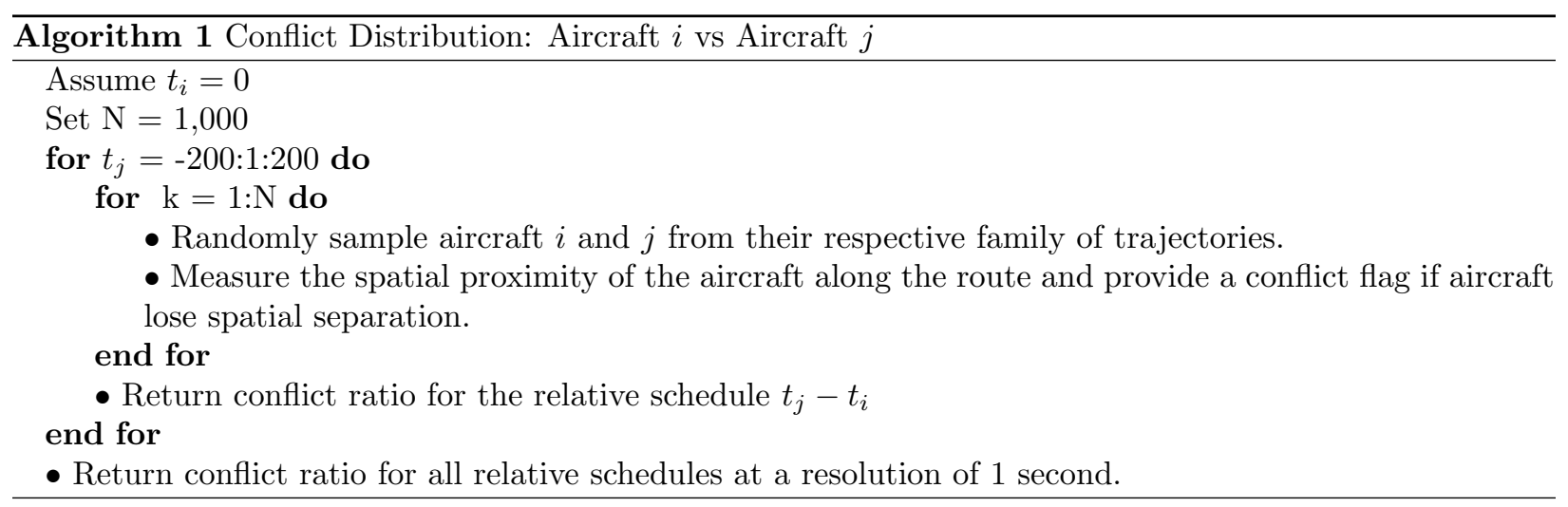

which ensures that if arriving aircraft $j$ follows arriving aircraft $i$ at merge node P2 they should be separated by a minimum of $\delta_{i j}^{*}$, else the constraint is automatically satisfied.

For all departing aircraft $i \in D$ and arriving aircraft $j \in A$ we introduce the constraint

$$
a_{i j}^{L B}+a_{i j}^{U B}=1 \quad \forall i \in A, j \in D
$$

where $a_{i j}^{L B}$ is a binary variable that is 1 for departing aircraft $i$ and arriving aircraft $j$ if we release the arriving aircraft into the ramp area to the left of the lower bound of the conflict with departing aircraft $j$, see Fig. 5, else $a_{i j}^{L B}=0$. Similarly $a_{i j}^{U B}$ is a binary variable that is 1 for departing aircraft $i$ and arriving aircraft $j$ if we release the arriving aircraft into the ramp area to the right of the upper bound of the conflict with departing aircraft $j$.
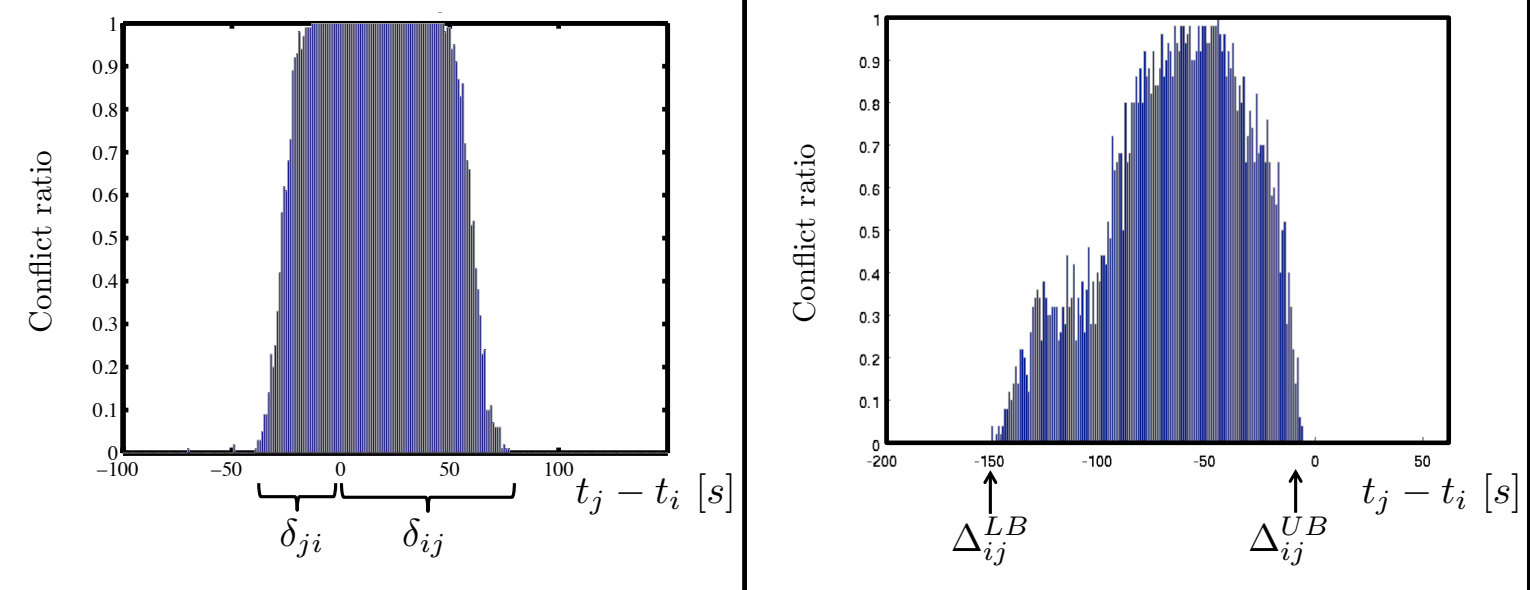

Figure 5. Conflict distributions computed using Algorithm 1. Left: Conflict distribution for CLT departure B6( $i)$ VS. CLT departure B8 $(j)$. The terminal time of departing aircraft $i$ is fixed at time $t_{i}=0$ and the terminal time for departing aircraft $j$ is given by the value on the horizontal axis. Right: Conflict distribution for CLT departure C9 $(i)$ VS. CLT arrival B6 $(j)$. The terminal time of departing aircraft $i$ is fixed at time $t_{i}=0$ and the release time for arriving aircraft $j$ is given by the value on the horizontal axis.

For all departing aircraft $i \in D$ and arriving aircraft $j \in A$ we have the separation constraint

$$
a_{i j}^{L B}\left(t_{j}-t_{i}-\Delta_{i j}^{L B}\right) \leq 0 \quad \forall i \in D, j \in A
$$

when $a_{i j}^{L B}=1$ this ensures the scheduled time $t_{j}$ that we release arriving aircraft $j$ into the ramp area is a minimum of $\Delta_{i j}^{L B}$ prior to the scheduled terminal time $t_{i}$ that we require departing aircraft $i$ to arrive at merge node P1.

For all departing aircraft $i \in D$ and arriving aircraft $j \in A$ we also have the separation constraint

$$
a_{i j}^{U B}\left(t_{j}-t_{i}-\Delta_{i j}^{U B}\right) \geq 0 \quad \forall i \in D, j \in A
$$


when $a_{i j}^{U B}=1$ this ensures the scheduled time $t_{j}$ that we release arriving aircraft $j$ into the ramp area is a minimum of $\Delta_{i j}^{U B}$ after the scheduled terminal time $t_{i}$ that we require departing aircraft $i$ to arrive at merge node P1.

This formulation solves for the optimal schedule that minimizes the summation of aircraft hold time while also ensuring conflict free trajectories. The program is in the form of a Mixed Integer Quadratic Program due to quadratic constraints (11), (13), and (15-16). In order to pass this program to a MILP solver we linearize the quadratic constraints as

$$
\begin{aligned}
t_{j}-t_{i}-\delta_{i j}+\left(1-z_{i j}\right) M \geq 0 & \forall i, j \in D \\
t_{j}-t_{i}-\delta_{i j}^{*}+\left(1-Z_{i j}^{*}\right) M \geq 0 & \forall i, j \in A \\
t_{j}-t_{i}-\Delta_{i j}^{L B}-\left(1-a_{i j}^{L B}\right) M \leq 0 & \forall i \in D, j \in A \\
t_{j}-t_{i}-\Delta_{i j}^{U B}+\left(1-a_{i j}^{U B}\right) M \geq 0 & \forall i \in D, j \in A
\end{aligned}
$$

where the constant $\mathrm{M}$ is chosen to be sufficiently large. Constraint (17) is a linear separation constraint that replaces the quadratic constraint (11), constraint (18) is a linear separation constraint replacing the quadratic constraint (13), constraint (19) is a linear separation constraint that replaces the quadratic constraint (15), and constraint (20) is a linear separation constraint replacing the quadratic constraint (16). After formulating the program as a MILP we solve for the optimal time schedule by utilizing the Gurobi Optimizer ${ }^{27}$ solver. $^{2}$

\section{MILP Example Solutions}

Here we provide example solutions of the MILP. In order to output an optimal schedule the first thing we do is select the set of departing and arriving aircraft. For this paper we define a scenario as a set of three departing aircraft and two arriving aircraft. After the departing (arriving) aircraft are defined, we sample the parameters $\alpha_{i}\left(\beta_{i}\right)$ for each aircraft $i$ from the uniform distribution defined as $\alpha_{i}\left(\beta_{i}\right) \sim \mathcal{U}(0,100)$.

For departing aircraft $i$ the parameter $t_{S 0}^{i}\left(t_{F 0}^{i}\right)$ is determined by the robot experiment data and is defined by the earliest (latest) feasible push back time such that $t_{i}=0$ is enforced. Using the values $t_{S 0}^{i}$ and $t_{F 0}^{i}$, the push back bounds $t_{S}^{i}$ and $t_{F}^{i}$ for any given scheduled spot time $t_{i}$ can be computed as $t_{S}^{i}=t_{i}+t_{S 0}^{i}$ and $t_{F}^{i}=t_{i}+t_{F 0}^{i}$. All other necessary parameters for the MILP are computed from the conflict distributions as previously mentioned. The output of the program is a schedule of merge node times $t_{i}$ that minimizes the summation of aircraft hold time. Furthermore, the model provides the feasible push back windows for each departing aircraft, see Table 1.

\begin{tabular}{|l|c|c|c|c|c|}
\hline Aircraft & $\alpha_{i}\left(\beta_{i}\right)$ & Merge Node Time: $t_{i}$ & Aircraft Hold & $t_{S}$ & $t_{F}$ \\
\hline \hline Arrival: B8 & 87 & 248 & 161 & NA & NA \\
\hline Arrival: C7 & 2 & 2 & 0 & NA & NA \\
\hline Departure: B6 & 50 & 198 & 0 & 50 & 83 \\
\hline Departure: B10 & 5 & 124 & 0 & 5 & 31 \\
\hline Departure: C9 & 47 & 253 & 55 & 104 & 133 \\
\hline
\end{tabular}

Table 1. An optimal solution for CLT example scenario 1 containing three departing aircraft at gates B6, B10, and C9 and two arriving aircraft that terminate their trajectories at gates B8 and C7. The earliest available time $\alpha_{i}\left(\beta_{i}\right)$ that aircraft $i$ is available to initiate their trajectory is sampled from the uniform distribution defined as $\alpha_{i}\left(\beta_{i}\right) \sim \mathcal{U}(0,100)$. For departing trajectories the feasible window in time to initiate their push back is defined by the values $t_{s}$ and $t_{f}$.

The example scenario that we consider in Table 1 is defined by three departing aircraft at gates B6, B10, and C9 and two arriving aircraft that terminate their trajectories at gates B8 and C7. For the given scenario, the optimal schedule and the associated aircraft hold times are dependent upon the set of sampled earliest available times $\alpha_{i}\left(\beta_{i}\right)$. For a different set of earliest available times $\alpha_{i}\left(\beta_{i}\right)$, the optimal schedule and aircraft hold times can be quite different. For a given scenario (set of departing and arriving aircraft), we would like to understand how our scheduling MILP performs under a variety of different sets of earliest available times.

In order to understand the overall performance of the MILP we fix the scenario, compute the optimal schedule for many different sets of earliest available times, and then we average the hold time of each aircraft 


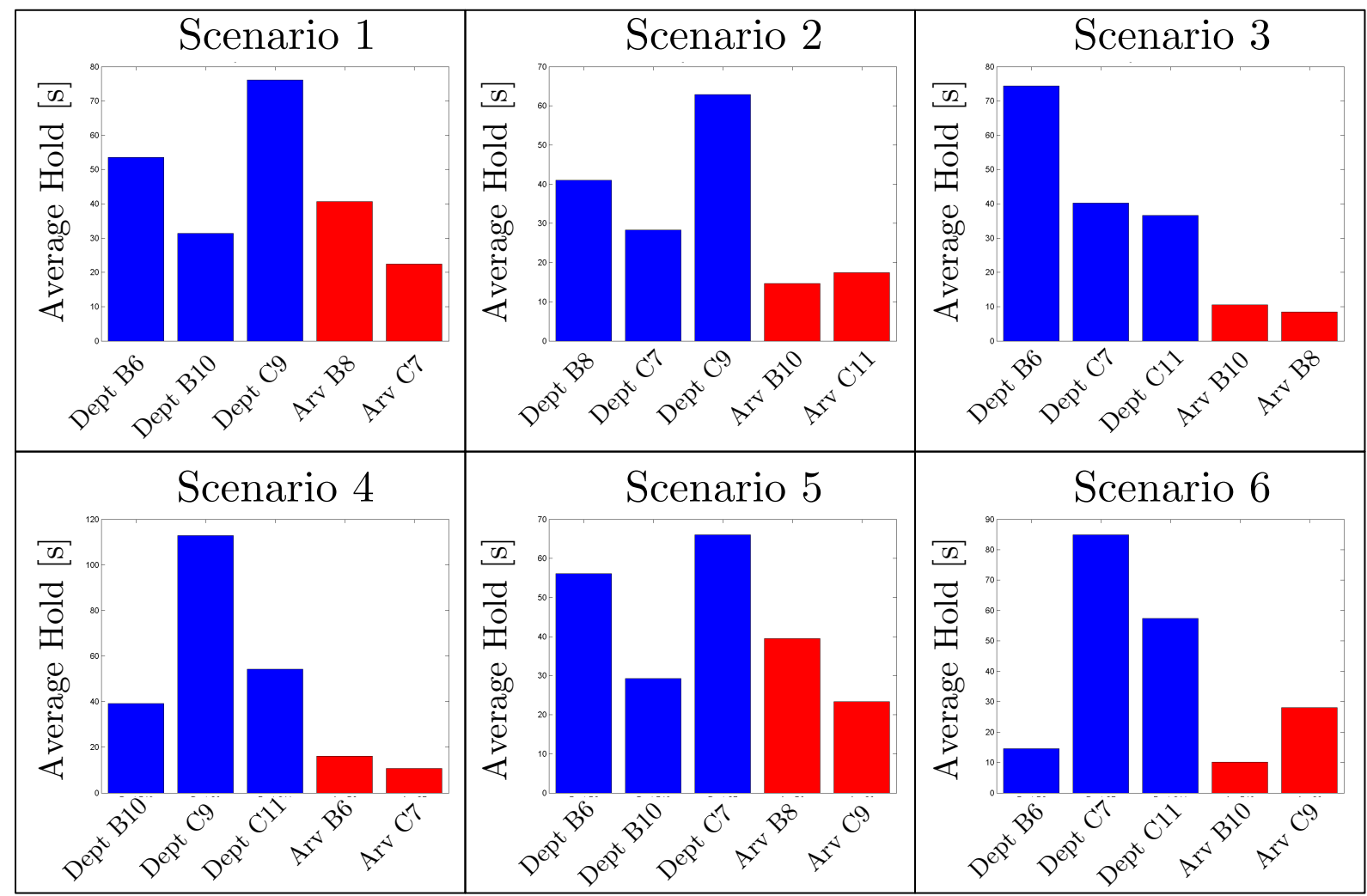

Figure 6. Top: Example scenario 1, 2, and 3 from left to right. Bottom: Example scenario 4, 5 and 6 from left to right. The average hold time for various departing (blue) and arriving (red) aircraft operating within the CLT center alley. Each figure is defined by a different scenario of three departing and two arriving aircraft. The earliest available time $\alpha_{i}\left(\beta_{i}\right)$ that aircraft $i$ is available to initiate their trajectory is sampled from the uniform distribution defined as $\alpha_{i}\left(\beta_{i}\right) \sim \mathcal{U}(0,100)$. Solutions for each scenario are computed for 300 randomly sampled sets of earliest available input parameters and the average hold time for each aircraft within the six different example scenarios is plotted.

within the scenario, see Fig. 6. In the upper left most figure (scenario 1), the average hold time for departing aircraft B6, B10 and C9 are shown with blue bars while the average hold time for arriving aircraft B8 and $\mathrm{C} 7$ are shown with red bars. We apply the same analysis of averaging the hold time over many different sampled sets of earliest available times and apply it to five additional scenarios (sets of departing and arriving aircraft), see Fig. 6.

\section{Discussion}

In this work we used sampling methods to build sets of feasible ramp area aircraft trajectories. These sampled trajectories were used to compute conflict distributions among aircrafts $i$ and $j$. Once the conflict distributions were computed, we estimated minimum-time separation constraints between any two aircraft $i$ and $j$. Using these separation constraints we formulated a MILP and solved the optimal taxiway scheduling problem defined by objective (7).

The separation constraints that we used in the MILP formulation are conservative in nature. The schedules are constrained to provide the minimum-time separation at the taxiway spot that ensures a zero ratio of conflict. Using the conservative conflict constraints, we solved for the average hold time for six different scenarios defined by three departing aircraft and two arriving aircraft.

For schedules that have a non-zero ratio of conflict, future work will consider techniques that can eliminate the conflicts between aircraft $i$ and $j$ by shrinking the push back time windows. Using these techniques, the throughput of the conservative schedule can be improved upon. We would also like to improve upon the time data that we use as input to the stochastic model of aircraft trajectories. Moving forward we will use 
data from NASA Ames' FutureFlight Central $^{28}$ real-time human in the loop simulations.

\section{References}

${ }^{1}$ Gilbo, E. P., "Airport capacity: representation, estimation, optimization," IEEE Transactions on Control Systems Technology, Vol. 1, No. 3, 1993, pp. 144-154.

${ }^{2}$ Jung, Y., Hoang, T., Montoya, J., Gupta, G., Malik, W., L., T., and Wang, H., "Performance Evaluation of a Surface Traffic Management Tool for Dallas/Fort Worth International Airport," 9th USA/Europe Air Traffic Management Research and Development Seminar, 2011, pp. 1-10.

${ }^{3}$ Pesic, B., Durand, D., and Alliot, J. M., "Aircraft ground traffic optimisation using a genetic algorithm," Genetic and Evolutionary Computation Conference (GECCO), San Francisco, CA, 2001.

${ }^{4}$ Aliot, J. M., Gotteland, J.-P., Durand, N., and Page, E., "Aircraft ground traffic optimization," 4th International Air Traffic management RED Seminar, Santa Fe, NM, 2001.

${ }^{5}$ Nemhauser, G. L. and Wolsey, L. A., Integer and combinatorial optimization, Wiley-Interscience, New York, NY, USA, 1988.

${ }^{6}$ García, J., Berlanda, A., Molina, J., and Casar, J., "Optimization of airport ground operations integrating genetic and dynamic flow management algorithms," AI Commun, Vol. 18, 2005, pp. 143-164.

${ }^{7}$ Herrero, J. G., Berlanda, A., Molina, J. M., and Casar, J. R., "Methods for operations planning in airport decision support systems," Appl Intell, Vol. 22, 2005, pp. 183-206.

${ }^{8}$ Smeltink, J. W., Sooner, M. J., de Waal, P. R., and van der Mei, R. D., "An optimisation model for airport taxi scheduling," INFORMS Annual Meeting, 2004.

${ }^{9}$ Marín, A., "Airport management: taxi planning," Ann Oper Res, Vol. 143, 2007, pp. 191-202.

${ }^{10}$ Roling, P. C. and Visser, H. G., "Optimal airport surface traffic planning using mixed-integer linear programming," Int J Aerospace Eng, Vol. 2008, 2008, pp. 1-12.

${ }^{11}$ Clare, G. L. and Richards, A. G., IEEE Transactions on Intelligent Transportation Systems, Vol. 12, No. 4, 2011, pp. 1000-1013.

${ }^{12}$ Malik, W., Gupta, G., and Jung, Y. C., "Managing departure aircraft release for efficient airport surface operations," Proceedings of the AIAA Guidance, Navigation, and Control (GNC) Conference, Toronto, Canada, 2010.

${ }^{13}$ Malik, W., Gupta, G., and Jung, Y. C., "Spot Release Planner: Efficient Solution for Detailed Airport Surface Trffic Optimization," 12th AIAA Aviation Technology, Integration, and Operations (ATIO) Conference, Indianapolis, Indiana, 2012.

${ }^{14}$ Gupta, G., Malik, W., and Jung, Y. C., "A Mixed Integer Linear Program for Airport Departure Scheduling," 9th AIAA Aviation Technology, Integration, and Operations Conference (ATIO), Hilton Head, South Carolina, 2009.

${ }^{15}$ Gupta, G., Malik, W., and Jung, Y. C., "Incorporating Active Runway Crossings in Airport Departure Scheduling," AIAA Guidance, Navigation, and Control Conference (GNC), Toronto, Ontario Canada, 2010.

${ }^{16}$ Balakrishnan, H. and Jung, Y., "A framework for coordinated surface operations planning at DFW international airport," AIAA Guidance, Navigation, and Control Conference (GNC), Reston, VA, 2007.

${ }^{17}$ Rathinam, S., Montoya, J., and Jung, Y., "An optimization model for reducing aircraft taxi times at the DFW international airpiort," 26th International Conference of the Aeronautical Sciences, Ancorage, AK, 2008.

${ }^{18}$ Anderson, R. and Milutinović, D., "An approach to optimization of airport taxiway scheduling and traversal under uncertainty," Proceedings of the Institution of Mechanical Engineers, Part G: Journal of Aerospace Engineering, Vol. 227, No. 2, February 2013, pp. 273-284.

${ }^{19}$ Atkins, S., Brinton, C., and Jung, Y., "Implication of Variability in Airport Surface Operations on 4-D Trajectory Planning," Proceedings of the 8th AIAA Aviation Technology, Integration, and Operations (ATIO) Conference, 2008.

${ }^{20}$ Ravizza, S., Atkin, J. A. D., Maathuis, M. H., and Burke, E. K., "A combined statistical approach and ground movement model for improving taxi time estimations at airports." JORS, Vol. 64, No. 9, 2013, pp. 1347-1360.

${ }^{21}$ Gupta, G., Malik, W., and Jung, Y. C., "Effect of Uncertainty on Deterministic Runway Scheduling," 11th AIAA Aviation Technology, Integration, and Operations (ATIO) Conference, Virgina Beach, VA, 2010.

${ }^{22}$ Coupe, W. J., Milutinović, D., Malik, W., Gupta, G., and Jung, Y., "Robot Experiment Analysis of Airport Ramp Area Time Constraints," AIAA Guidance, Navigation, and Control Conference (GNC), Boston, MA, 2013.

23 "Website of EPFL Education Robot," URL http://www.e-puck.org/.

24 "Website of Boeing 747-400 Airplane Characteristics," URL http://www.boeing.com/commercial/airports/747.htm.

${ }^{25}$ Milutinović, D., "Utilizing Stochastic Process for Computing Distributions of Large-Size Robot Population Optimal Centralized Control," 10th International Symposium on Distributed Autonomous Robotic Systems (DARS), Lausanne, Swizterland, 2010.

${ }^{26}$ Gillespie, D. T., "Exact Stochastic Simulation of Coupled Chemical Reactions," The Journal of Physical Chemistry, Vol. 81, 1977.

27 "Website of Gurobi Optimizer Mathematical Program Solver," URL http://www.gurobi.com/.

28 "Website of FutureFlight Central," URL http://www.aviationsystemsdivision.arc.nasa.gov/facilities/ffc/index. shtml. 